\title{
Regulation of RAB22A by mir-193b inhibits breast cancer growth and metastasis mediated by exosomes
}

\author{
LIANG SUN ${ }^{1,2}$, MIAO HE $^{3}$, NING XU ${ }^{1}$, DA-HAI XU ${ }^{1}$, YAACOV BEN-DAVID ${ }^{4,5}$, \\ ZHAO-YING YANG ${ }^{2 *}$ and YOU-JUN LI ${ }^{1 *}$ \\ ${ }^{1}$ Department of Human Anatomy, College of Basic Medical Sciences, Jilin University, Changchun, Jilin 130021; \\ ${ }^{2}$ Department of Breast Surgery, China-Japan Union Hospital of Jilin University, Changchun, Jilin 130033; \\ ${ }^{3}$ Department of Anesthesia, The Second Hospital of Jilin University, Changchun, Jilin 130022; \\ ${ }^{4}$ State Key Laboratory for Functions and Applications of Medicinal Plants, Guizhou Medical University, \\ Guiyang, Guizhou 550025; ${ }^{5}$ The Key Laboratory for Traditional Chinese Medicine of Guizhou Province and \\ Chinese Academy of Science, Guiyang, Guizhou 550014, P.R. China
}

Received July 20,2018; Accepted September 7, 2018

DOI: 10.3892/ijo.2018.4571

\begin{abstract}
Breast cancer is one of the main types of cancer affecting the health of females worldwide. Despite improvements in therapeutic approaches, cancer patients succumb to the disease due to metastasis itself, rather than the primary tumor from which metastases arise, emphasizing the need for the better understanding of the biological bases that contribute to disease progression. RAB22A, a member of the proto-oncogene RAS family, plays an important role in the formation, trafficking and metabolism of exosomes, and is associated with the occurrence and development of multiple human cancers. In this study, we demonstrate that the upregulation of $R A B 22 A$ is associated with breast cancer progression and lymph node metastasis. We identified a signature of $R A B 22 A$ and miR-193b that exhibited a negative association in metastatic as opposed to the surrounding normal cells, and $R A B 22 A$ was identified as the target gene of miR-193b. While RAB22A was found to regulate exosomes-mediated breast cancer cell proliferation, invasion and migration, these biological characteristics were diminished in the breast cancer cells in which the $R A B 22 A$ gene was knocked down or in the cells in which the exosomes
\end{abstract}

Correspondence to: Professor You-Jun Li, Department of Human Anatomy, College of Basic Medical Sciences, Jilin University, 126 Xinmin Boulevard, Changchun, Jilin 130021, P.R. China E-mail: youjun08@hotmail.com

Professor Zhao-Ying Yang, Department of Breast Surgery, ChinaJapan Union Hospital of Jilin University, 126 Xiantal Boulevard, Changchun, Jilin 130033, P.R. China

E-mail: zhaoyingyang@163.com

*Co-senior authorship

Key words: breast cancer, microRNA, gene expression, cell communication, exosomes biosynthesis were dissolved by proteinase $\mathrm{K} / \mathrm{RNase}$ treatment. On the whole, the findings of this study demonstrate the critical role that miR-193b plays in the regulation of RAB22A-mediated exosome function during cancer growth and metastasis, which may have significant implications on cancer therapy.

\section{Introduction}

Breast cancer is a major cause of mortality among women worldwide (1). Despite much progress being made in therapeutic approaches, including early detection, surgery, radiation, as well as endocrine and anti-HER-2 therapies, cancer patients succumb to the disease due to metastasis, which is incurable $(2,3)$. Therefore, the better understanding of the biological pathways that contribute to disease progression and the development of novel therapeutic modalities is of paramount importance.

MicroRNAs (miRNAs or miRs), are small, single-stranded, endogenous, non-coding RNA molecules, 20-23 nucleotides in length, which play an essential role in the regulation of gene expression at the post-transcriptional level. In several malignant tumors, such as breast, lung, hepatocellular and pancreatic cancer, significant differences have been found in the expression profiles of miRNAs compared to normal tissues (4-7). These miRNAs regulate the expression of tumor suppressor genes and/or oncogenes. For example, the overexpression of miR-506 has been shown to inhibit the expression of tumor suppressor gene, IQ motif containing GTPase activating protein 1 (IQGAPI) (8); conversely, the lower expression or absence of miR-9 has been shown to block the downregulation of the HER-2 oncogene, leading to breast cancer progression (9).

In our pervious study, we observed that miR-193b was significantly downregulated in breast cancer cell lines, which promoted tumor cell proliferation, migration and invasion; $R A B 22 A$ was also identified as a potential downstream target of this miRNA (10). RAB22A is a small GTPase, belonging to the RAB protein family, RAB5 subfamily (11), which mainly 
presents in early endosomes, Golgi bodies and late endosomes. RAB proteins are involved in the regulation of vesicular traffic and exosome formation (12). Studies have found that the RAB5 subfamily (including RAB5, RAB21, RAB22A and $\mathrm{RAB} 22 \mathrm{~B}$ ) is mainly involved in the endocytosis, transport and metabolism of growth factor receptors, and may thus be associated with cancer progression (13-15). In different cell lines, RAB22A is known as an endosomal-associated protein, involved in exocrine vehicle formation $(16,17)$. At the plasma membrane of MDA-MB-231 cells, RAB22A has been shown to be a component of microvesicles. In breast cancer cells, the knockdown of the $R A B 22 A$ gene has been shown to decrease hypoxia-induced exocrine vehicle formation and the cell invasive ability (17).

Exosomes are a type of exocrine vehicle, ranging in diameter from 30 to $100 \mathrm{~nm}$, originating from the intracellular body. Exosomes contain membrane-anchored receptors, adhesion molecules, signaling proteins, active oncogenes and nucleic acids. Exosomes can alter the biological behavior of these recipient cells by transferring their cargo to recipient cells and are now considered to be key players in cell-to-cell communications. For example, cancer cells release exosomes carrying proteins, lipids and nucleic acids that promote tumor progression, reprogram tumor microenvironment and modulate immune response (18).

Our previous study on miR-193b demonstrated its relevance in cancer development, progression and metastasis (10). However, the mechanisms through which miR-193b interacts with its targets, leading to cancer development/progression, remain largely unknown. In this study, we thus investigated the role of miR-193b in the regulation of its target gene, $R A B 22 A$, and in the alteration of the exosomal biosynthesis pathway by altering the biological behavior of recipient cells. The findings of this study may aid in the development of novel targeted therapies.

\section{Materials and methods}

Human tissues. We collected 23 groups of samples (20 used for RT-qPCR and 3 for western blot analysis) we with breast cancer lesions (including tumor and metastatic lymph node) and their matched adjacent normal breast tissues from patients with both breast cancer and axillary lymph node metastases at the Clinic of the Breast Surgery Department of China-Japan Union Hospital affiliated to Jilin University (Jilin, China). All the clinical procedures were approved by the Committees of Clinical Ethics of China-Japan Union Hospital of Jilin University (2017022218, Jilin, China). Written informed consents were obtained from individual or guardian participants.

Cell lines and transfection. All cell lines were purchased from the American Type Culture Collection (ATCC, Manassas, VA, USA), including human normal mammary epithelial MCF-10A cells were grown in DMEM/F12 Ham's Mixture supplemented with $5 \%$ horse serum (Gemini Bio, Woodland, CA, USA), EGF $20 \mathrm{ng} / \mathrm{ml}$, insulin $10 \mu \mathrm{g} / \mathrm{ml}$, hydrocortisone $0.5 \mathrm{mg} / \mathrm{ml}$ and cholera toxin $100 \mathrm{ng} / \mathrm{ml}$ (all from Sigma-Aldrich, St. Louis, MO, USA). The primary human breast cancer cell lines, MCF-7, MDA-MB-231 and MDA-MB-453, were cultured as previously described (10). The SK-BR-3, ZR-75 and T47D cells were grown in RPMI supplemented with $10 \%$ fetal bovine serum (FBS; Gibco/Thermo Fisher Scientific, Waltham, MA, USA); the MDA-MB-468 cells were grown in L-15 medium with $10 \%$ FBS. All cell lines were maintained at $37^{\circ} \mathrm{C}$ and $5 \% \mathrm{CO}_{2}$.

The synthetic mimic pre-miR-193b (stem-loop sequence: GUGGUCUCAGAAUCGGGGUUUUGAGGGCGAGAUGA GUUUAUGUUUUAUCCAACUGGCCCUCAAAGUCCCG CUUUUGGGGUCAU), the negative-scramble control RNAs (SC), pcDNA3.1-RAB22A ORF plasmid and blank pcDNA3.1 vectors were purchased from Ambion/Thermo Fisher Scientific. The MCF-7 or SK-BR-3 cells were seeded in 6-cm dishes until approximately $70 \%$ confluency, and Lipofectamine 2000 (Invitrogen/Thermo Fisher Scientific) was then added to the diluted miR and plasmid at a final concentration of $40 \mathrm{nmol} / 1$, followed by incubation for $20 \mathrm{~min}$ at room temperature, and was then added to the cell medium. The cells were then incubated at $37^{\circ} \mathrm{C}, 5 \% \mathrm{CO}_{2}$ for $6 \mathrm{~h}$. Subsequently, fresh medium was added. The cells were harvested $24 \mathrm{~h}$ later for used in RT-qPCR, western blot analysis and in vitro experiments.

Short hairpin RNA (shRNA) sequences were designed by Hanbio Biotechnology Co. Ltd. (HH20170612LLN-LV01, Shanghai, China) to target human RAB22A. After annealing, double strands of shRNA were inserted into lentiviral pHBLV-Scramble-Puro vector (Hanbio, Shanghai, China). This construct, named shRAB22A, and a negative-scramble control, named SC were used according to the manufacturer's instructions. In brief, $2 \times 10^{5}$ cells were incubated with $2 \times 10^{7}$ transducing units (TU) lentivirus and $8 \mu \mathrm{g}$ Polybrene (Hanbio) in $1 \mathrm{ml}$ cell medium at $37^{\circ} \mathrm{C}, 5 \% \mathrm{CO}_{2}$ for $4 \mathrm{~h}$. Subsequently, $1 \mathrm{ml}$ fresh medium was added for $24 \mathrm{~h}$ prior to changing back to normal media. Stable cell lines were generated after $48 \mathrm{~h}$ by using puromycin (Hanbio) selection.

RT-qPCR. Human tissues were obtained and analyzed. The MCF-10A, MCF-7, MDA-MB-231, MDA-MB-453, SK-BR-3, ZR-75, T47D and MDA-MB-468 were cultured in indicated medium. For mRNA expression, total RNA was purified using TRIzol reagent (Invitrogen/Thermo Fisher Scientific) according to the manufacturer's instructions. Following quantitation using Synergy HT (BioTek, Highland Park, Illinois, USA), $1 \mu \mathrm{g}$ of total RNA was reverse transcribed using SuperScript III Reverse Transcriptase (Invitrogen/Thermo Fisher Scientific) according to the manufacturer's instructions. The cDNA (100 ng) was added to the RT-qPCR mixture containing SYBR Premix ExTaq II (Takara Bio Inc., Kusatsu, Shiga, Japan) and the appropriate primers. All RT-qPCR analyses were performed using the ABI PRISM 7900 Sequence Detection System (Applied Biosystems, Foster City, CA, USA). The cycling parameters are as follows: Initial melting at $95^{\circ} \mathrm{C}$ for $30 \mathrm{sec}$, followed by 40 cycles of $95^{\circ} \mathrm{C}$ for $5 \mathrm{sec}, 57^{\circ} \mathrm{C}$ for $30 \mathrm{sec}$ and $68^{\circ} \mathrm{C}$ for $30 \mathrm{sec}$. The relative fold change in RNA expression was calculated using the $2^{-\triangle \Delta \mathrm{Cq}}$ method (19), GAPDH was used as an endogenous control for normalization. The expression of miRNA was conducted by using TaqMan MicroRNA Assay (Applied Biosystems) specific for hsa-miR-193b and RNU44. Prior to RT, the RNA extracted using the mir-Vana kit (Ambion/Thermo Fisher Scientific). Firstly, $5 \mu 1$ total RNA with $3 \mu \mathrm{l}$ specific primers were added to TaqMan MicroRNA 
Reverse Transcription kit (Applied Biosystems), incubated at $16^{\circ} \mathrm{C}$ for $30 \mathrm{~min}, 42^{\circ} \mathrm{C}$ for $30 \mathrm{~min}, 85^{\circ} \mathrm{C}$ for $5 \mathrm{~min}$ and hold in $4^{\circ} \mathrm{C}$. Then, the products were subsequently amplified by incubated at $95^{\circ} \mathrm{C}$ for $10 \mathrm{~min}$, followed by 40 cycles of $95^{\circ} \mathrm{C}$ for $15 \mathrm{sec}$ and $60^{\circ} \mathrm{C}$ for $1 \mathrm{~min}, \mathrm{RNU} 44$ was used as an endogenous control for normalization. The primer sequences used in this study were as follows: $R A B 22 A$ forward (5'-3'), ttgtagttgccattgcagga and reverse (5'-3'), aggctgtcttcggagtttga; $G A P D H$ forward $\left(5^{\prime}-3^{\prime}\right)$, gtctcctctgacttcaacagcg and reverse $\left(5^{\prime}-3^{\prime}\right)$, accacctgttgctgtagccaa.

Cell proliferation and clonogenic assay. The SK-BR-3 or MCF-7 cells cultured for the indicated periods of time (0-5 days), and cell viability was detected using the cell counting kit-8 (CCK-8) according to the manufacturer's instructions (Beyotime Institute of Biotechnology, Shanghai, China) and read using a microplate reader (Synergy HT, BioTek), at $450 \mathrm{~nm}$.

The clonogenicity of a single cell was detected by colony assay. Following treatment with $0.25 \%$ trypsin, the SK-BR-3 or MCF-7 cells were collected and adjusted to a concentration of 300 cells/Petri dish, and $2 \mathrm{ml}$ of pre-heated culture medium were then added. The cells were cultured at $37^{\circ} \mathrm{C}$ with $5 \% \mathrm{CO}_{2}$ for 2 weeks. After the colonies were visible to the naked eye, the cells were washed twice with phosphate-buffered saline (PBS), fixated with 4\% paraformaldehyde for 15 min before Giemsa (Sigma-Aldrich) staining for $10 \mathrm{~min}$ at room temperature and the number of colonies was counted.

Transwell assays. The SK-BR-3 cells were maintained in RPMI supplemented with $10 \%$ FBS, and $1 \times 10^{5}$ cells were plated on BD BioCoat MATRIGEL Invasion Chambers with Matrigel and control inserts with polyethylene terephthalate membrane (BD Biosciences, San Jose, CA, USA) to assess the cell invasive and migratory abilities as previously described $(10,20)$.

Exosome isolation and purification. Exosomes were isolated from the supernatant either of the SK-BR-3 or MCF-7 cell culture media. In brief, $2 \times 10^{6}$ cells were plated in a Petri dish with RPMI supplemented with $10 \%$ FBS and $1 \%$ penicillin/streptomycin. The following day, the medium was changed to exosome-free RPMI supplemented with $10 \%$ exosome-depleted FBS. The culture medium was collected until the cells reached $70-80 \%$ confluence and exosomes were isolated by ultracentrifugation with a rotor (L8-80M; Beckman, Brea, CA, USA) at the centrifugal force of $110,000 \mathrm{x} g$ for $70 \mathrm{~min}$. Exosomes were re-washed in PBS at $110,000 \mathrm{x} \mathrm{g}$ for $70 \mathrm{~min}$ to eliminate contaminating proteins, then re-suspended in $100 \mu \mathrm{l}$ PBS and immediately tested or stored at $-80^{\circ} \mathrm{C}$ for further analysis. TSG101 and HSP70 levels were detected by western blot analysis as exosome markers.

To dissolve exosome components, exosomes were treated $\left(37^{\circ} \mathrm{C}, 60 \mathrm{~min}\right.$ ) with $500 \mu \mathrm{g} / \mathrm{ml}$ proteinase K (Sigma-Aldrich) dissolved in RNase-free water, followed by heat inactivation of the protease $\left(60^{\circ} \mathrm{C}, 10 \mathrm{~min}\right)$ and incubation $\left(37^{\circ} \mathrm{C}, 10 \mathrm{~min}\right)$ with $2 \mu \mathrm{g} / \mathrm{ml}$ protease-free RNase A (Sigma-Aldrich).

Western blot analysis. For western blot analysis, aliquots of total protein extracts $(25 \mu \mathrm{g})$ from human tissues, cells or exosomes were loaded and separated by SDS-PAGE (15\%), and samples were transferred onto PVDF membranes (Millipore, Billerica, MA, USA). The membranes were then blocked in $5 \%$ bovine serum albumin and incubated at $4{ }^{\circ} \mathrm{C}$ overnight with specific primary antibodies. The secondary antibodies (diluted 1:2,000), anti-rabbit or anti-mouse IgG antibodies (cat. no. 7074 or 7076) from Cell Signaling Technology (Beverly, MA, USA), were added followed by incubation at room temperature for $2 \mathrm{~h}$, and the detected signals were visualized using BeyoECL Plus (Beyotime Institute of Biotechnology, Haimen, Jiangsu, China), as recommended by the manufacturer. Densitometry was performed using ImageJ software (Bundled with 64-bit Java for Windows, version 1.8, Bethesda, MD, USA). The densitometry value for each sample was normalized against the value for GAPDH or $\beta$-actin to obtain the intensities for $\mathrm{RAB} 22 \mathrm{~A}$ reported in the figures. To examine the concentrations of proteins in the lysates, the following antibodies were used: RAB22A (1:500, cat. no. 16181-1-AP; Proteintech, Rosemont, IL, USA), TSG101 (1:500, cat. no. sc-7964), HSP70 (1:500, cat. no. sc-32239), Calnexin (1:500, cat. no. sc-23954), GAPDH (1:1,000, cat. no. sc-47724) (all from Santa Cruz Biotechnology, Santa Cruz, CA, USA), TFIIB (1:1,000, cat. no. 4169; Cell Signaling Technology) and $\beta$-actin (1:5,000, cat. no. A5441; Sigma-Aldrich).

Co-culture experiments. A total of 1x106 SK-BR-3 or MCF-7 cells were plated in a Petri dish in exosome-depleted culture medium and then supplemented with the indicated exosomes (20 $\mu \mathrm{g} / \mathrm{ml})$, every day for 2 days. Subsequently, $2 \times 10^{4}$ cells were seeded for the indicated days and then subjected to cell density assay. For the Transwell assay, after priming with the exosomes, $1 \times 10^{5}$ cells were plated on the chambers to assess the cell invasive and migratory abilities.

Fluorescence microscopy analysis. The SK-BR-3 cells grown on coverglass slips were incubated for $1 \mathrm{~h}$ with the fluorescent probe DiO (Vybrant DiO Cell-Labling Solution from Invitrogen/Thermo Fisher Scientific) labeled exosomes in RPMI at $37^{\circ} \mathrm{C}$. The cells were then washed with PBS to remove unbound labeled exosomes, fixed with $4 \%$ paraformaldehyde for $15 \mathrm{~min}$ and stained nuclei with DAPI (4',6-diamidino-2-phenylindole, Invitrogen/Thermo Fisher Scientific) at room temperature for 5 min and subsequently imaged with a Zeiss LSM 510 Axiovert 200M microscope (Carl Zeiss Inc., Oberkochen, Germany) to produce confocal-like images.

Statistical analysis. Experimental cultures were set up in triplicate and each experiment was repeated at least 3 times. Data are presented as the means \pm standard error unless otherwise indicated. Unpaired two-tailed student t-tests or analysis of variance (ANOVA) were used to determine the P-values for differences between samples. One-way ANOVA, followed by the LSD post hoc test was used to compare mean differences between 3 or more groups. Two-way ANOVA, followed by Dunnett's or Sidak's multiple comparisons test was used for multiple comparisons. Means with P-values $<0.05$ were considered statistically significant. The ratio of the protein band intensities relative to that of GAPDH or $\beta$-actin, the quantification of colonies in clonogenic assay and cells in Transwell assays was carried out for each sample using ImageJ software (Bethesda, MD, USA). 
A

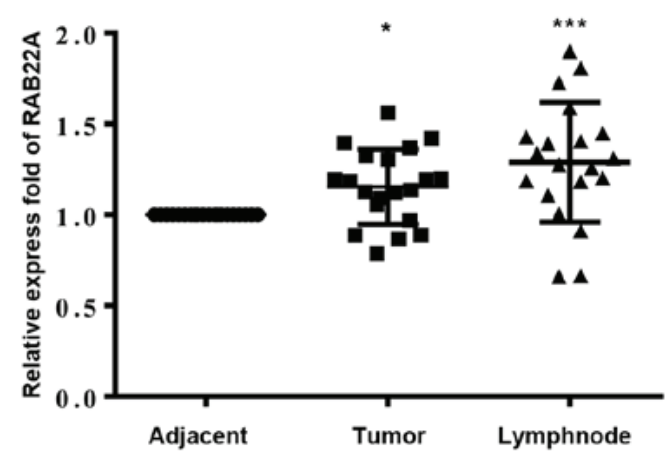

B

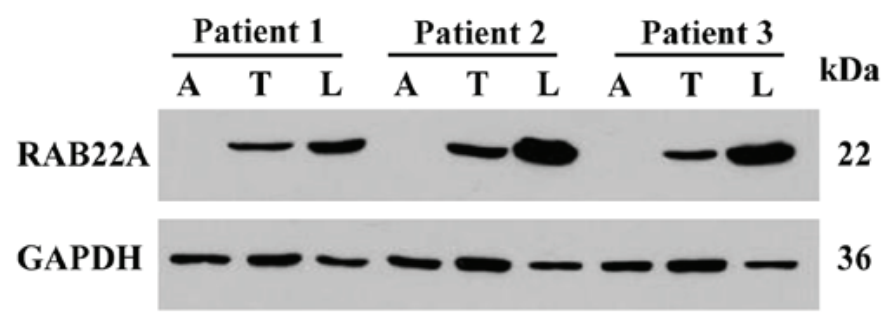

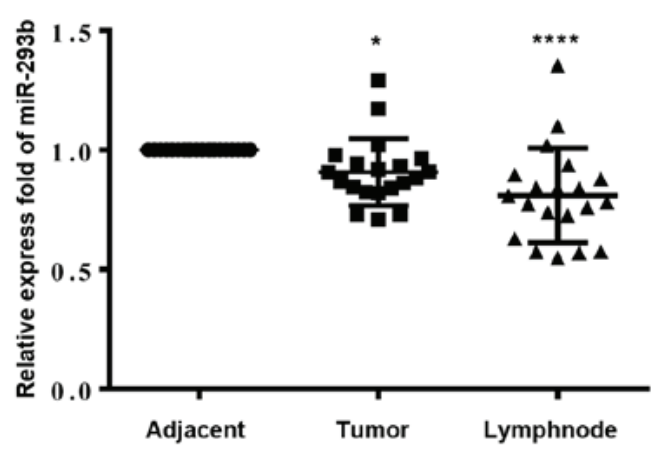

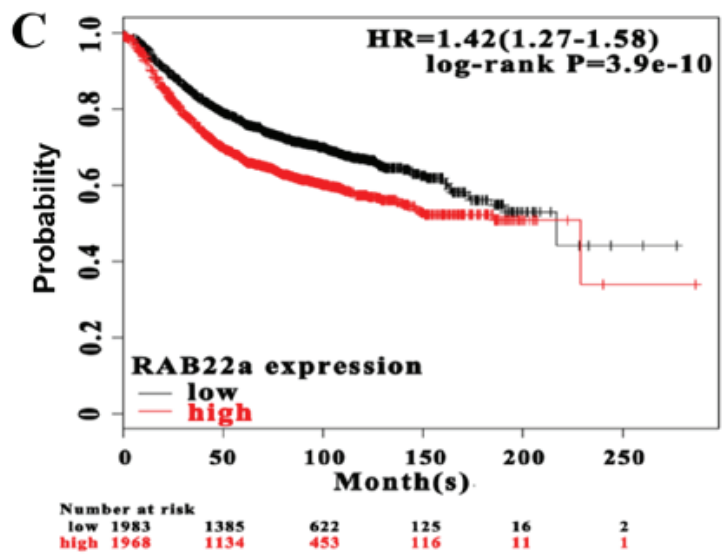

Figure 1. Upregulation of $R A B 22 A$ is accompanied by tumor formation and lymph node metastasis. (A) Fold change of $R A B 22 A$ and miR-193b gene expression in tumor and metastatic lymph node tissue relative to adjacent tissue $(n=20)$. (B) Western blot analysis of RAB22A protein expression in breast cancer tissues (lanes labeled ' $\mathrm{T}$ ') and metastatic lymph node tissues (lanes labeled ' $\mathrm{L}$ ') compared to that in the corresponding adjacent normal breast tissues (lanes labeled 'A'); GAPDH was used as a loading control. (C) Kaplan-Meier plots for RAB22A mRNA expression in breast cancer. Log-rank P-values and hazard ratio (HR; 95\% confidence interval in parentheses) are shown. ${ }^{*} \mathrm{P}<0.05,{ }^{* * *} \mathrm{P}<0.001$ and ${ }^{* * * * *} \mathrm{P}<0.0001$ vs. adjacent normal tissues, determined by one-way ANOVA followed by Fishers' LSD test.

We used KM Plotter (http://kmplot.com/analysis/) to determine the prognostic values of $R A B 22 A$ in breast cancer (21). Gene expression data and survival information of 3,951 breast cancer patients downloaded from GEO by KM Plotter. Cancer patients were divided into the high and low expression groups by the median values of $R A B 22 A \mathrm{mRNA}$ expression, and the 2 groups were then compared by log-rank test followed by Cox proportional hazards regression, and a Kaplan-Meier plot was drawn. The number of cases, median values of mRNA expression level, HR, 95\% CI and log-rank P-value were extracted from the KM plotter webpage.

\section{Results}

Upregulation of RAB22A is associated with tumor production and lymph node metastasis. To further examine the role of miR$193 \mathrm{~b}$ and $R A B 22 A$ in predicting breast cancer proliferation and metastasis (10), the expression levels of these genes were analyzed simultaneously by RT-qPCR using 20 groups of samples with breast cancer lesions and their matched adjacent normal breast tissues. In this analytical approach, we identified a signature of $R A B 22 A$ and miR-193b, exhibiting a negative association between the tumor and peritumoral tissues (Fig. 1A). Whereas the gene expression level of miR-193b was decreased, that of $R A B 22 A$ was increased in the cancer lesions when compared with the adjacent normal tissues. RAB22A protein extraction from a random selection of 3 groups of samples revealed that its level was increased in the cancer lesions, particularly in the metastatic tissues with lymph node metastasis, compared to the corresponding adjacent normal tissues (Fig. 1B). Gene expression data and survival information of 3,951 breast cancer patients downloaded from GEO, patients were divided into high and low expression group by the median values of $R A B 22 A$ mRNA expression. The Kaplan-Meier survival curve was used to confirm the prognostic power of RAB22A mRNA (Fig. 1C), which revealed that a lower expression of $R A B 22 A$ was associated with a longer survival time of the patients. These results confirm our previous observation showing a similar negative association between $R A B 22 A$ and miR-193b in breast cancer cell lines (10).

$R A B 22 A$ is the regulatory target gene of $m i R-193 b$. A negative association between miR-193b and $R A B 22 A$ expression was also observed in the normal breast epithelial MCF-10A cells compared to the other human breast cancer cell lines, such as MDA-MB-453, MDA-MB-231, SK-BR-3, MCF-7, MDA-MB-468, ZR-75 and T47D (Fig. 2A, B and E). This result supports our previous observation suggesting $R A B 22 \mathrm{~A}$ as a downstream target of miR-193b in breast cancer (10). To further confirm this observation, in this study, miR-193b mimic was transfected into the MCF-7 and SK-BR-3 cell lines. The results revealed that miR-193b overexpression (Fig. 2C) markedly downregulated $R A B 22 A$ gene expression, as detected by RT-qPCR (Fig. 2D) and western blot analysis (Fig. 2F). 

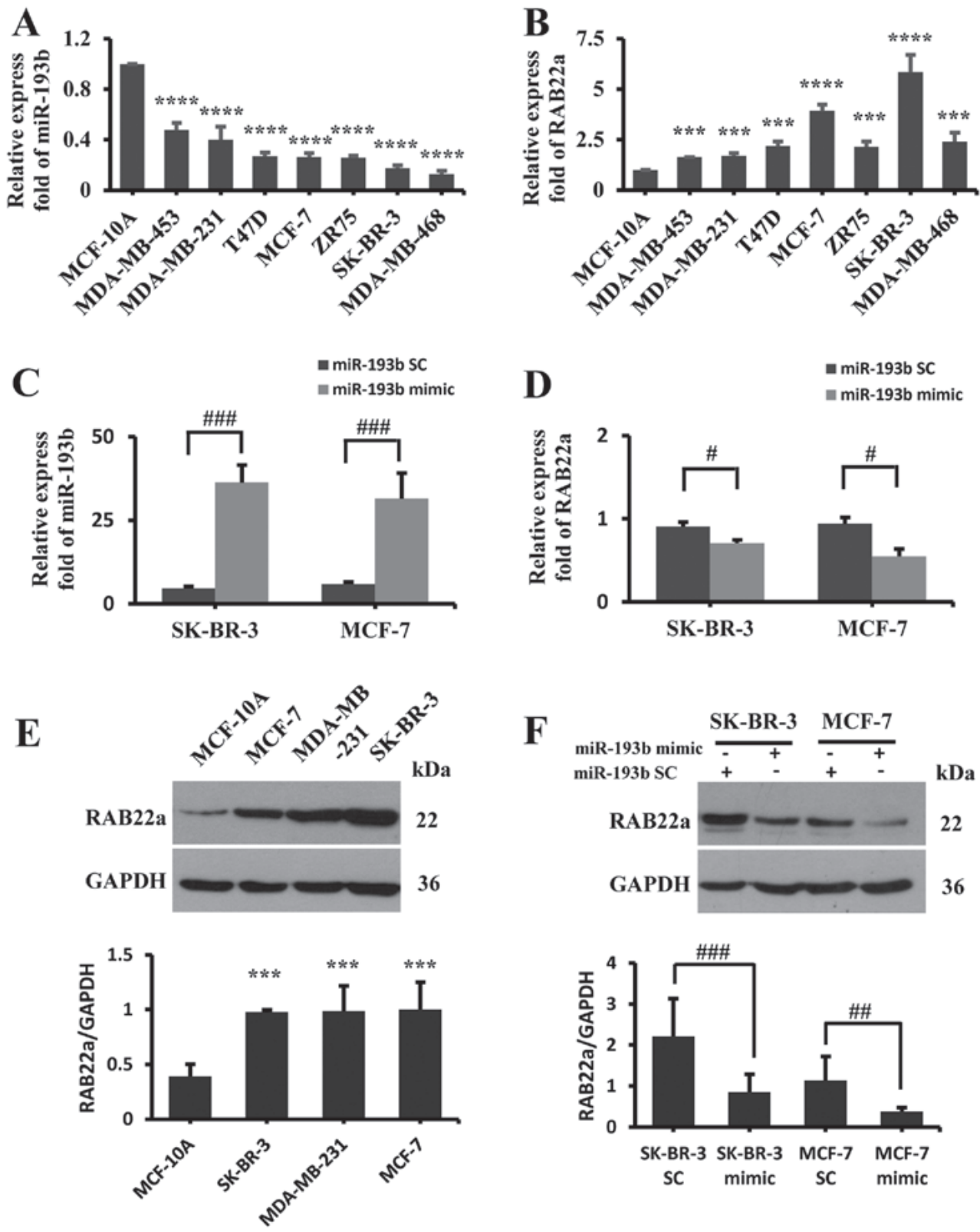

Figure 2. RAB22A is the regulatory target of miR-193b. (A and B) RT-qPCR detection of miR-193b and RAB22A gene expression levels in the indicated breast cancer cell lines. MCF-10A cells (immortalized normal breast epithelial cells) were used as a control. (C) RT-qPCR detection of miR-193b expression in miR-193b mimic-transfected MCF-7 and SK-BR-3 cells. (D) RT-qPCR detection of RAB22A expression in miR-193b mimic-transfected MCF-7 and SK-BR-3 cells. (E) Western blot analysis of RAB22A protein expression in the indicated cell lines; GAPDH was used as loading control. (F) Western blot analysis of RAB22A expression following transfection of the MCF-7 and SK-BR-3 cells with miR-193b mimic. (E and F) The band density of the blots was quantified by densitometry relative to GAPDH and presented as graphs on the bottom panels. SC, scramble control. ${ }^{\#} \mathrm{P}<0.05,{ }^{\# \#} \mathrm{P}<0.01$ and ${ }^{\# \# \#} \mathrm{P}<0.001 \mathrm{vs}$. SC, determined by the Student's t-test; ${ }^{* * *} \mathrm{P}<0.001$ and ${ }^{* * * * *} \mathrm{P}<0.0001$ vs. MCF-10A cells, determined by one-way ANOVA followed by Fishers' LSD test.

RAB22A knockdown inhibits the cell proliferative ability of breast cancer cells. To demonstrate the role of $R A B 22 A$ in breast cancer, this gene was knocked down in both the SK-BR-3 and MCF-7 cells. The results of RT-qPCR analysis revealed that the decreased in $R A B 22 A$ mRNA expression was close to $70 \%$ of the levels observed in the control cells (Fig. 3A). While the mRNA levels of RAB22A were similar between the SK-BR-3 and MCF-7 cells, the decrease in the RAB22A protein level was more evident in the SK-BR-3 than in the MCF-7 cells (Fig. 3B). The knockdown of RAB22A or the overexpression of miR-193b by transfection with miR-193b mimic in the MCF-7 and SK-BR-3 cells resulted in a significant reduction in proliferation compared to the control, after 5 days in culture (Fig. 3C). To further confirm whether the tumor-suppressive role of miR-193b is mediated by $R A B 22 A$, a gain-of-function analysis was performed. Firstly, we transfected the MCF-7 and SK-BR-3 cells with either negative-scramble control or mimic pre-miR-193b along with either pcDNA3.1 or pcDNA3.1-RAB22A to detect the protein level of RAB22A (Fig. 3D). We found that the overexpression of $R A B 22 A$ completely abolished the effects of miR-193b on the proliferation and invasion of the SK-BR-3 cells (Fig. 3E). To examine the long-term effects of $R A B 22 A$ gene knockdown on the MCF-7 and SK-BR-3 cells, these cells and their corresponding controls were cultured at low density for 2 weeks. Quantification of the colonies formed 
A

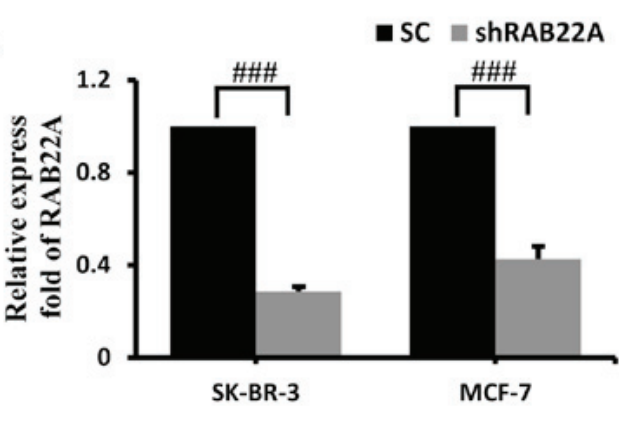

C
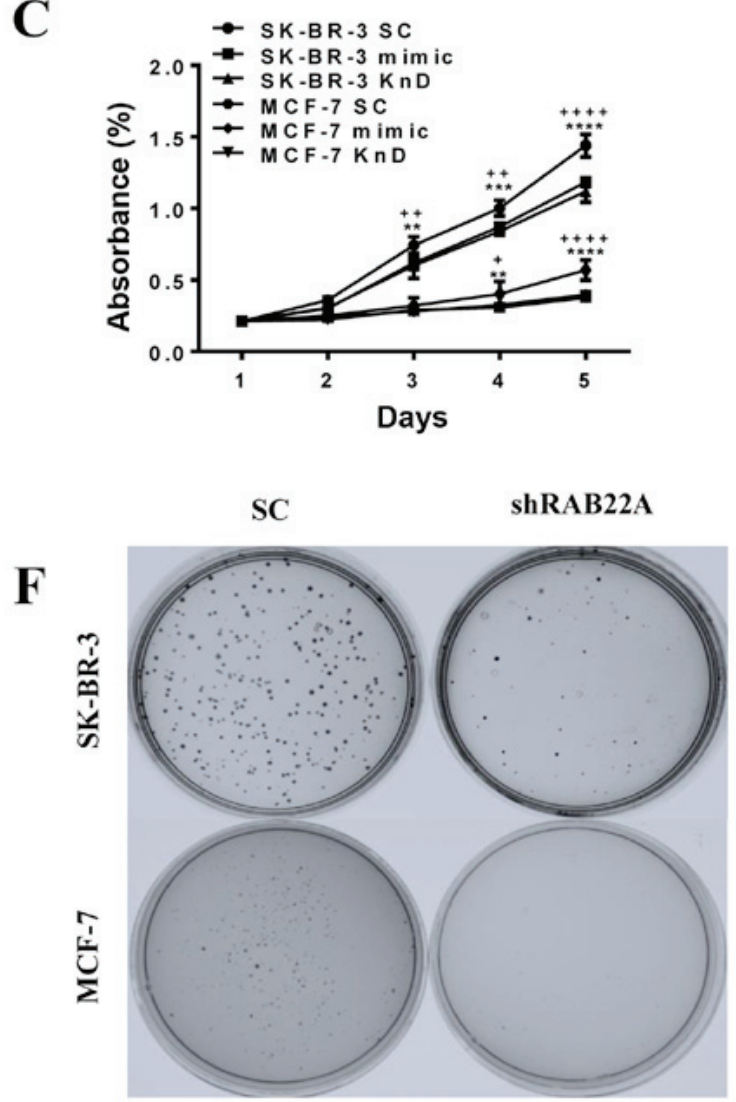

B

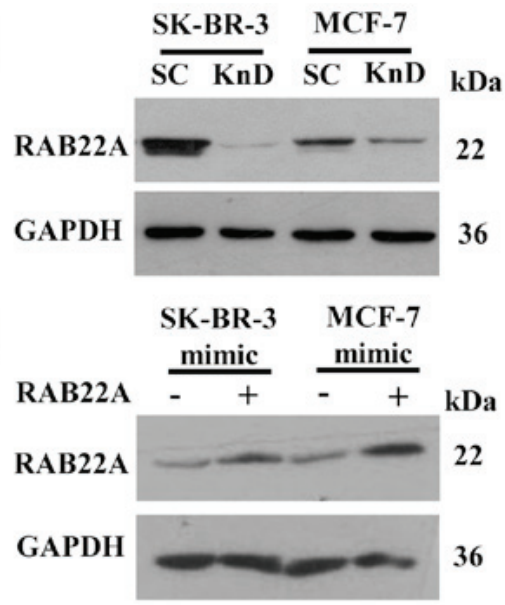

E

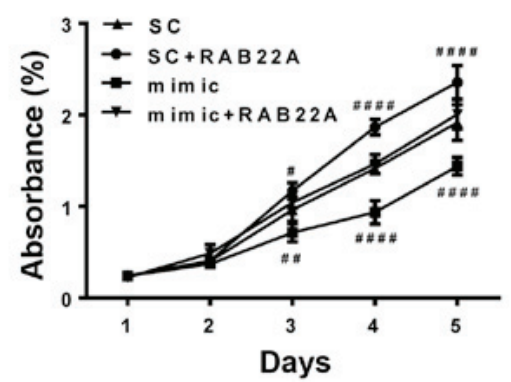

G

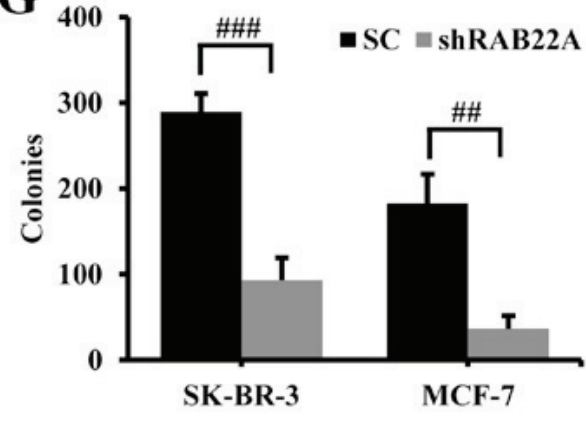

Figure 3. Analysis of the effects of $R A B 22 A$ gene knockdown. (A and B) SK-BR-3 and MCF-7 cells were infected with shRAB22A (KnD) or the scrambled control (SC). The relative expression of $R A B 22 A$ in the related groups was detected by RT-qPCR and western blot analysis. (C) The indicated cells were cultured for 0-5 days and the proliferation rate was measured by CCK-8 assay. (D) The protein level of RAB22A was measured in the SK-BR-3 and MCF-7 cells transfected with miR-193b mimic along with either pcDNA3.1 or pcDNA3.1-RAB22A. (E) The effect of the miR-193b mimic on the proliferative potential of SK-BR-3 cells was detected in the presence of pcDNA3.1-RAB22A treatment via CCK-8 assay. (F) Images of colonies formed were obtained after the plating of the indicated cells for 2 weeks followed by Giemsa staining. (G) Quantification of colonies formed from the SK-BR-3 and MCF-7 cells infected

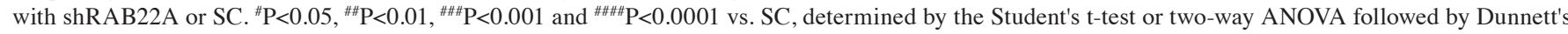
multiple comparisons test. ${ }^{* *} \mathrm{P}<0.01,{ }^{* * *} \mathrm{P}<0.001$ and ${ }^{* * * *} \mathrm{P}<0.0001$ vs. KnD; ${ }^{+} \mathrm{P}<0.05,{ }^{++} \mathrm{P}<0.01$ and ${ }^{++++} \mathrm{P}<0.0001$ vs. mimic, determined by two-way ANOVA followed by Dunnett's multiple comparisons test.

from the single cells revealed that the knockdown of $R A B 22 A$ reduced the number of colonies when compared to the control cells (Fig. 3F and G).

The $R A B 22 A$ regulation of exosomes mediates the migratory and invasive ability of the $S K-B R-3$ cells. Consistent with the known involvement of $R A B 22 A$ in exosome function and trafficking (17), the knockdown of $R A B 22 A$ or overexpression of miR-193b by transfection with miR-193b mimic in the SK-BR-3 cells decreased the level of exosomes in the cancer cells (Fig. 4A). Western blot analysis also revealed the reduced expression of the exocrine marker proteins, namely the multi-vesicular body formation protein TSG101 and the exosomal cargo protein HSP70 in the exosomes from the cells in which $R A B 22 A$ was knocked down and the cells transfected with the miR-193b mimic; however, this was not found in the total protein isolated from these cells. However, no changes in the expression of TFIIB and Calnexin were detected in both the exosomes and extracts from the total cells (Fig. 4B). To further confirm whether the RAB22Arelated exosomes affect cell growth, exosomes secreted from the SK-BR-3 cells were added back to the culture medium of 


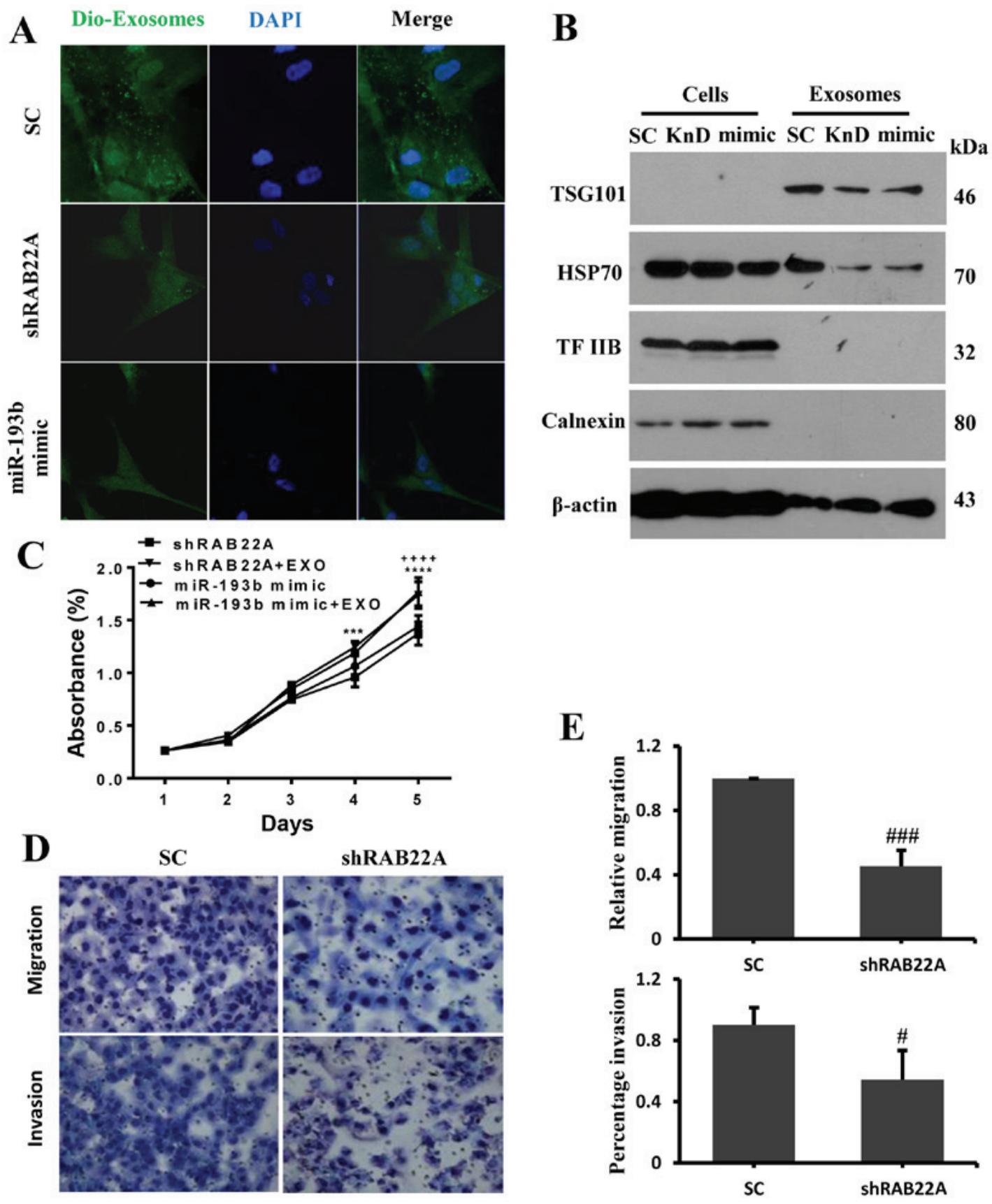

Figure 4. RAB22A regulates the exosome-mediated migratory and invasive ability of SK-BR-3 cells. (A) Images of DiO-labeled exosomes, DAPI-stained nuclei (blue) and the merged image, shown by confocal microscopy. (B) Western blot analysis of the indicated proteins in SK-BR-3 cells following transfection with miR193b mimic, shRNA against the RAB22A gene (Knd), or the scramble control (SC). $\beta$-actin was used as a loading control. (C) The effect of exosomes on the proliferative potential of SK-BR-3 cells in which $R A B 22 A$ was knocked down or transfected with the miR-193b mimetic was detected the cells in which exosomes were added back to the culture by CCK-8 assays. (D) Polyethylene terephthalate membrane or Matrigel assay was used to detect the migratory or invasive ability of the SK-BR-3 cells in which $R A B 22 A$ was knocked down. (E) Quantitative analysis of the above experiment. Relative migration was calculated by comparison with cells infected with the negative control. The percentage invasion was calculated based on the number of cells which had invaded through the Matrigel insert, divided by the number of cells which had migrated through the control insert. EXO, exosomes. "P<0.05 and ${ }^{\# \# \# ~} \mathrm{P}<0.001 \mathrm{vs}$. SC, determined by the Student's t-test. ${ }^{* * * *} \mathrm{P}<0.001$ and ${ }^{* * * * *} \mathrm{P}<0.0001$ vs. shRAB22A; ${ }^{++++} \mathrm{P}<0.0001$ vs. mimic, determined by two-way ANOVA followed by Sidak's multiple comparisons test.

the cells in which $R A B 22 A$ was knocked down and the cells transfected with miR-193b mimic; increased cell growth was observed (Fig. 4C). To examine whether RAB22A affects cell migration and invasion to another organ, as observed in patients via lymph node metastasis, Transwell assays were performed. The results shown in Fig. 4D and E revealed that $R A B 22 A$ knockdown significantly inhibited cell migration and invasion.
To further confirm the role of $R A B 22 A$ in proliferation and invasion, exosomes were prepared from the supernatant of the SK-BR-3 and MCF-7 cells in which RAB22A was knocked down, or transfected with the miR-193b mimic or scrambled (SC) control cells, as depicted in Fig. 5A. A total of $2 \times 10^{4}$ cells from both the SK-BR-3 and MCF-7 cells in which $R A B 22 A$ was knocked down and the controls were plated in a Petri dish in exosome-depleted culture medium, and their 
A
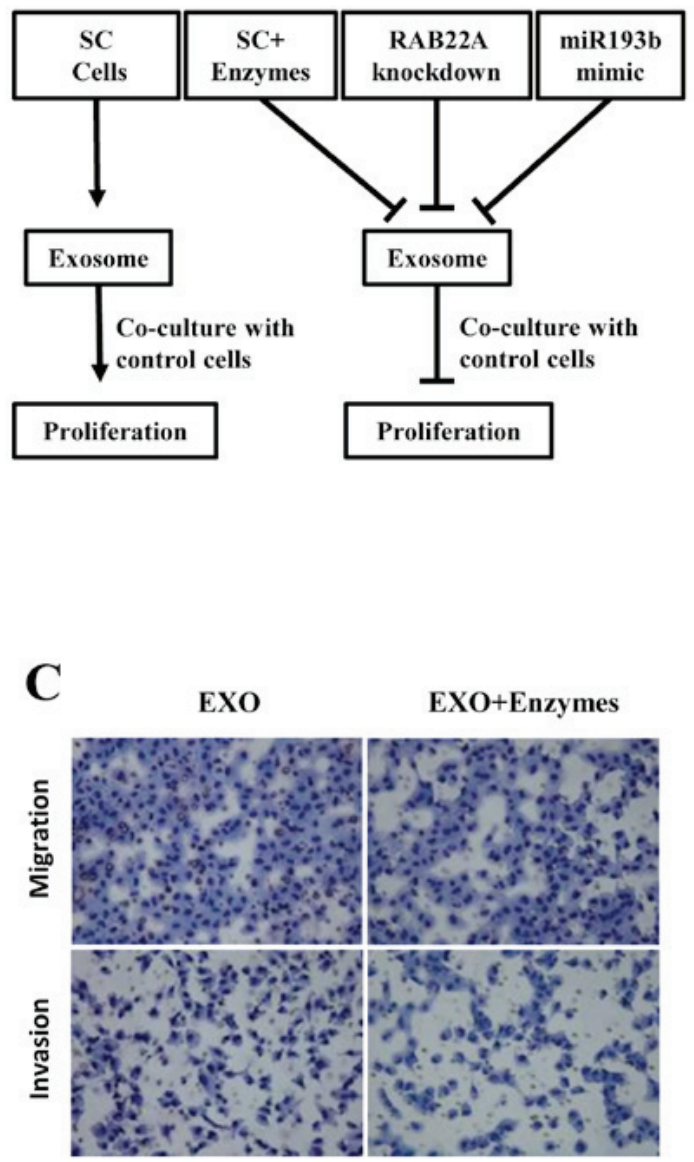
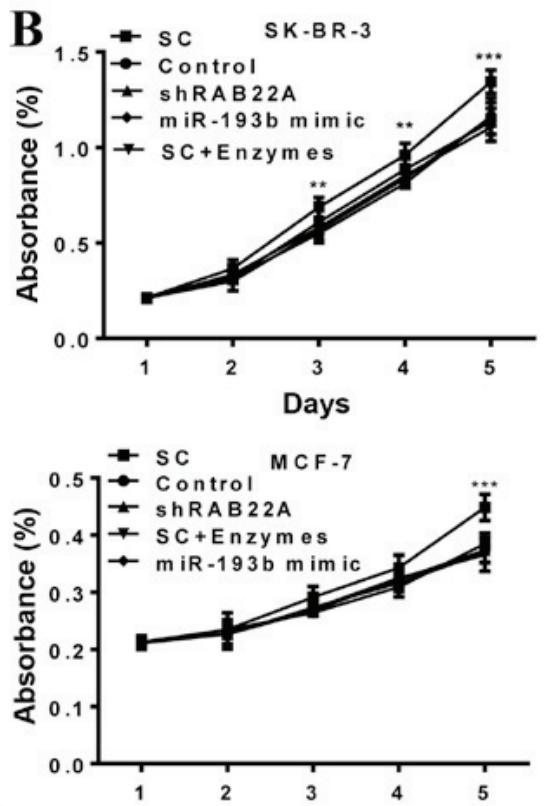

D
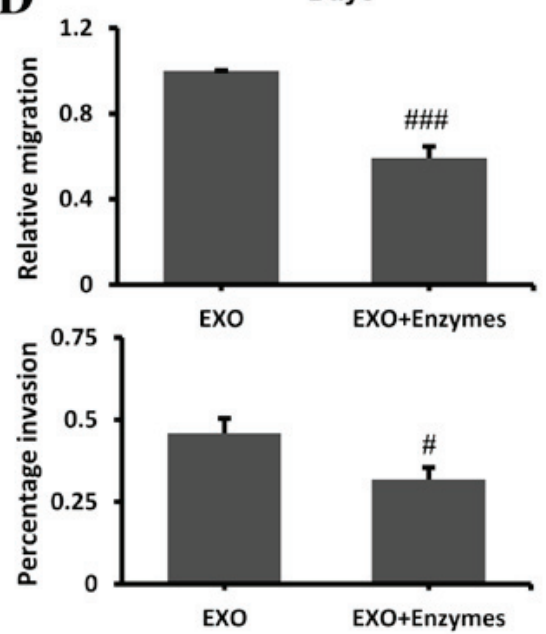

Figure 5. Exosomes lacking RAB22A diminish breast cancer cell proliferation, invasion and migration. (A) SK-BR-3 and MCF-7 cells were infected with shRAB22A as a control. Schematic diagram of experiments using MCF-7 and SK-BR-3 cells transfected with miR-193b mimic, shRAB22A or scramble control (SC) with exosome culture. (B) Indicated exosomes were co-cultured with control cells for 0-5 days and proliferation was detected by CCK-8 assay. (C) Images of SK-BR-3 cells in which $R A B 22 A$ was knocked down cells and examined by Transwell assays with exosomes isolated from SK-BR-3 cell supernatants (EXO) treated with or without enzymes. (D) Quantitation of the above experiment. ${ }^{\#} \mathrm{P}<0.05$ and ${ }^{\# \# \prime} \mathrm{P}<0.001$ vs. EXO, determined by the Student's t-test. ${ }^{* *} \mathrm{P}<0.01$ and ${ }^{* * *} \mathrm{P}<0.001$ vs. Control, determined by two-way ANOVA followed by Dunnett's multiple comparisons test.

cultures were supplemented with the isolated exosomes for the indicated days. A greater cell proliferation was observed following co-culture with the exosomes generated from the cells transfected with the scramble control (SC group), but not from the cells in which $R A B 22 A$ was knocked down or the cells transfected with the miR-193b mimic. Treatment of the cells in the SC group with proteinase K/RNase (to dissolve the exosomes), also reduced the proliferative ability of these cells to similar levels observed with $R A B 22 A$ knockdown and transfection with miR-193b mimic (Fig. 5B). To examine whether exosome production affects cell migration and invasion to other organs, exosomes extracted from the SK-BR-3 cells were harvested and treated with or without proteinase K/RNase, then added to the culture of SK-BR-3 cells in which $R A B 22 A$ was knocked down in the Transwell assay. The results shown in Fig. 5C and D revealed that the addition of exosomes not treated with proteinase $\mathrm{K} / \mathrm{RNa}$ se increased the migration and invasion of these cells compared to the addition of exosomes that were treated with the enzyme.

\section{Discussion}

In a previous study, we identified RAB22A as a target of miR-193b by luciferase reporter assay, that their expression levels were negatively associated in cell lines and tumors from breast cancer patients, particularly in tissues with lymph node metastasis, as opposed to the surrounding normal cells. We found that the knockdown of $R A B 22 A$ in breast cancer cells attenuated tumor growth and invasion, a process that was mediated through exosomal function. These results support the role of $R A B 22 A$ in tumor progression and invasion, and suggests that it may have therapeutic implications for breast cancer.

Recent studies have demonstrated that miRNAs play important roles in cancer growth and metastasis, including breast 
cancer, and are associated with clinical characteristics and outcomes $(10,22)$. Among these miRNAs, emerging evidence suggests that miR-193b acts as a tumor suppressor in various types of cancer, such as breast, pancreatic, esophageal, ovarian, prostate cancer and Ewing sarcomas, through the downregulation of proto-oncogenes, RAB22A, CCND1, KRAS, STMN1, Cyclin DI and ERBB4 (10,23-28). This study demonstrated that the reconstitution of miR-193b expression in a breast cancer cell line resulted in decreased cell proliferation, clonogenicity, migration and invasion, and RAB22A was confirmed as a direct target of this miRNA (10); the recovery of RAB22A completely abolished the tumor suppressive effects of miR-193b. We further demonstrated the association between the low expression of miR-193b and a higher production of $R A B 22 A$ in a sample of patients, which was associated with a poor prognosis.

Despite the higher expression of $R A B 22 A$ in tumor tissue, its role in breast cancer remains unclear. With increasing malignancy and a poor prognosis, $R A B 22 A$ is highly expressed in various types of cancer. For instance, $\mathrm{Su}$ et al demonstrated that the upregulation of $R A B 22 A$ promoted melanoma cell growth (29). Proliferative effects can be observed from gastric and colorectal cancer cells via the silencing miR-204-5p expression which also targets $R A B 22 A(30,31)$. Wang et al also analyzed the association between clinical data and $R A B 22 A$ in $>700$ cases of breast cancer and found that a high mRNA expression of $R A B 22 A$ was associated with a shortened overall survival time. They also found that RAB22A-coated extracellular vesicles promoted the infiltration and migration of breast cancer under hypoxic conditions (17). Thus, $R A B 22 A$ may be a good target for therapeutic intervention in breast cancer.

As a 'new way' of cell-to-cell communication, extracellular vesicles within exosomes transmit special signals (including cell proliferation, migration and infiltration activities) between cells and tissues (32). Although exosomes can be secreted by a variety of cell types, the number and content of the exosomes are closely related to the physiological state of the parental cell $(33,34)$. A growing number of studies have indicated that exosomes are related to the occurrence and progression of tumor cells (35-37), which can regulate immune response, promote tumor angiogenesis, invasion, and metastasis, and even act directly on other tumor or non-tumor cells (38). Studies have also demonstrated that the level of exosomes released by cells (particularly tumor cells) under pathological conditions is significantly increased, and the components of exosomes differ from those under normal physiological conditions, which not only emphasizes the parental cell precision adjustment to the contents of exosomes, but also suggests their important role in tumor formation and development (39). For example, under hypoxic conditions, breast cancer cells exhibit an increased secretion of exosomes by the hypoxia-inducible factor (HIF)-1 and exosomes secreted by squamous cell carcinoma cells contain higher levels of angiogenesis-related proteins, such as RAB22A that play important role in this process $(17,40)$. However, the role of exosomes the modulation of the immune response, tumor microenvironment reprogramming and metastasis, to reprogram recipient cells in breast cancer, remains undefined. In this study, the amounts of exosomes secreted by the SK-BR-3 cells were reduced following the knockdown of $R A B 22 A$ or transfection with pre-miR-193b mimic, that also caused the downregulation of the $R A B 22 A$ gene, resulting in diminished migratory and infiltrative abilities of the cancer cells. The re-addition of the exosomes extracted from the supernatant of SK-BR-3 cells abrogated the tumor suppressor role of miR-193b or the lack of the $R A B 22 A$ gene. Moreover, when exosomes extracted from supernatant of breast cancer cells were co-cultured with SK-BR-3 and MCF-7 cells in which $R A B 22 A$ was knocked down, the abilities of the cells to proliferate, infiltrate and migrate were significantly enhanced, but this ability was neutralized by treating the exosomes with protein and RNA degrading enzymes. These results suggested that exosome components, including proteins and RNAs, the expression of which is affected by miR-193b and $R A B 22 A$, were likely responsible for tumor growth. The unknown nature of these components, which play a key role in tumor growth and metastasis, suggests an interesting area of research that warrants further investigation. Overall, these results, suggest a role for $R A B 22 A$ in exosome secretion and function.

In conclusion, in this study, we demonstrate that oncogenic $R A B 22 A$, regulated by miR-193b, affects the exosome-mediated growth, invasion, and metastasis of the recipient breast cancer cells. $R A B 22 A$ may thus prove to be a novel target for breast cancer therapy.

\section{Acknowledgements}

The authors would like to thank Professor David Spaner (Department of Medicine, University of Toronto, ON, Canada) for providing helpful and critical comments during the preparation of this manuscript. The authors would also like to thank Professor Ling Zhang (Department of Medical Laboratory, The First Hospital of Jilin University, Jilin, China) for technical editing.

\section{Funding}

This study was supported by an NSFC (China) grant 81372456 (to YJL) and by the Foundation for Training Young Talents in Science and Technology of Jilin Province Health and Family Planning Commission 2017Q035 (to ZYY).

\section{Availability of data and materials}

All data generated or analyzed in this study are included in this manuscript.

\section{Authors' contributions}

LS initiated the experiment, analyzed data and wrote the manuscript. MH, NX, DHX and YBD provided support with experimental techniques. YBD also contributed to manuscript writing and revision. ZYY and YJL conceived and designed the study and supervised all the experiments. All authors have read and approved the final manuscript.

\section{Ethics approval and consent to participate}

All the clinical procedures were approved by the Committees of Clinical Ethics of China-Japan Union Hospital of Jilin University (2017022218, Jilin, China). Written informed consents were obtained from individual or guardian participants. 


\section{Patient consent for publication}

Not applicable.

\section{Competing interests}

The authors declare that they have no competing interests.

\section{References}

1. Siegel RL, Miller KD and Jemal A: Cancer statistics, 2018. CA Cancer J Clin 68: 7-30, 2018.

2. Howlader N, Noone A, Krapcho M, Miller D, Bishop K and Altekruse S: SEER Cancer Statistics Review, 1975-2015. National Cancer Institute, Bethesda, MD, 2015.

3. Chaffer CL and Weinberg RA: A perspective on cancer cell metastasis. Science 331: 1559-1564, 2011.

4. Kanno S, Nosho K, Ishigami K, Yamamoto I, Koide H, Kurihara H, Mitsuhashi K, Shitani M, Motoya M, Sasaki S, et al: MicroRNA-196b is an independent prognostic biomarker in patients with pancreatic cancer. Carcinogenesis 38: 425-431, 2017.

5. Lu M, Kong X, Wang H, Huang G, Ye C and He Z: A novel microRNAs expression signature for hepatocellular carcinoma diagnosis and prognosis. Oncotarget 8: 8775-8784, 2017.

6. Peng Z, Pan L, Niu Z, Li W, Dang X, Wan L, Zhang R and Yang S: Identification of microRNAs as potential biomarkers for lung adenocarcinoma using integrating genomics analysis. Oncotarget 8: 64143-64156, 2017.

7. Ahmadinejad F, Mowla SJ, Honardoost MA, Arjenaki MG, Moazeni-Bistgani M, Kheiri S and Teimori H: Lower expression of miR-218 in human breast cancer is associated with lymph node metastases, higher grades, and poorer prognosis. Tumour Biol 39: 1010428317698362, 2017.

8. Sun G, Liu Y, Wang K and Xu Z: miR-506 regulates breast cancer cell metastasis by targeting IQGAP1. Int J Oncol 47: 1963-1970, 2015.

9. Sun G, Sun L, Liu Y, Xing H and Wang K: Her-2 expression regulated by downregulation of miR-9 and which affects chemotherapeutic effect in breast cancer. Cancer Gene Ther 24: 194-202, 2017.

10. Yang Z, He M, Wang K, Sun G, Tang L and Xu Z: Tumor suppressive microRNA-193b promotes breast cancer progression via targeting DNAJC13 and RAB22A. Int J Clin Exp Pathol 7: 7563-7570, 2014

11. Delprato A and Lambright DG: Structural basis for Rab GTPase activation by VPS9 domain exchange factors. Nat Struct Mol Biol 14: 406-412, 2007.

12. Antonyak MA, Wilson KF and Cerione RAR: R(h)oads to microvesicles. Small GTPases 3: 219-224, 2012.

13. Mesa R, Magadán J, Barbieri A, López C, Stahl PD and Mayorga LS: Overexpression of Rab22a hampers the transport between endosomes and the Golgi apparatus. Exp Cell Res 304: 339-353, 2005.

14. Weigert R, Yeung AC, Li J and Donaldson JG: Rab22a regulates the recycling of membrane proteins internalized independently of clathrin. Mol Biol Cell 15: 3758-3770, 2004.

15. Kauppi M, Simonsen A, Bremnes B, Vieira A, Callaghan J, Stenmark H and Olkkonen VM: The small GTPase Rab22 interacts with EEA1 and controls endosomal membrane trafficking. J Cell Sci 115: 899-911, 2002.

16. Johnson DL, Wayt J, Wilson JM and Donaldson JG: Arf6 and Rab22 mediate T cell conjugate formation by regulating clathrinindependent endosomal membrane trafficking. J Cell Sci 130 : 2405-2415, 2017

17. Wang T, Gilkes DM, Takano N, Xiang L, Luo W, Bishop CJ, Chaturvedi P, Green JJ and Semenza GL: Hypoxia-inducible factors and RAB22A mediate formation of microvesicles that stimulate breast cancer invasion and metastasis. Proc Natl Acad Sci USA 111: E3234-E3242, 2014.

18. Ruivo CF, Adem B, Silva M and Melo SA: The Biology of Cancer Exosomes: Insights and New Perspectives. Cancer Res 77: 6480-6488, 2017.

19. Livak KJ and Schmittgen TD: Analysis of relative gene expression data using real-time quantitative PCR and the 2(- $\Delta \Delta C(T))$ method. Methods 25: 402-408, 2001.
20. Justus CR, Leffler N, Ruiz-Echevarria M and Yang LV: In vitro cell migration and invasion assays. J Vis Exp: Jun 1, 2014 (Epub ahead of print). doi: 10.3791/51046.

21. Györffy B, Lanczky A, Eklund AC, Denkert C, Budczies J, Li Q and Szallasi Z: An online survival analysis tool to rapidly assess the effect of 22,277 genes on breast cancer prognosis using microarray data of 1,809 patients. Breast Cancer Res Treat 123: 725-731, 2010

22. Li X, Zhang Y, Shi Y, Dong G, Liang J, Han Y, Wang X, Zhao Q, Ding J, Wu K, et al: MicroRNA-107, an oncogene microRNA that regulates tumour invasion and metastasis by targeting DICER1 in gastric cancer. J Cell Mol Med 15: 1887-1895, 2011.

23. Li J, Kong F, Wu K, Song K, He J and Sun W: miR-193b directly targets STMN1 and uPA genes and suppresses tumor growth and metastasis in pancreatic cancer. Mol Med Rep 10: 2613-2620, 2014.

24. Nyhan MJ, O'Donovan TR, Boersma AW, Wiemer EA and McKenna SL: MiR-193b promotes autophagy and non-apoptotic cell death in oesophageal cancer cells. BMC Cancer 16: 101, 2016.

25. Nakano H, Yamada Y, Miyazawa T and Yoshida T: Gain-offunction microRNA screens identify miR-193a regulating proliferation and apoptosis in epithelial ovarian cancer cells. Int J Oncol 42: 1875-1882, 2013.

26. Jin X, Sun Y, Yang H, Li J, Yu S, Chang X, Lu Z and Chen J: Deregulation of the MiR-193b-KRAS Axis Contributes to Impaired Cell Growth in Pancreatic Cancer. PLoS One 10: e0125515, 2015.

27. Kaukoniemi KM, Rauhala HE, Scaravilli M, Latonen L, Annala M, Vessella RL, Nykter M, Tammela TL and Visakorpi T: Epigenetically altered miR-193b targets cyclin D1 in prostate cancer. Cancer Med 4: 1417-1425, 2015.

28. Moore C, Parrish JK and Jedlicka P: MiR-193b, downregulated in Ewing Sarcoma, targets the ErbB4 oncogene to inhibit anchorage-independent growth. PLoS One 12: e0178028, 2017.

29. Su F, Chen Y, Zhu S, Li F, Zhao S, Wu L, Chen X and Su J: RAB22A overexpression promotes the tumor growth of melanoma. Oncotarget 7: 71744-71753, 2016.

30. Zhang B, Yin Y, Hu Y, Zhang J, Bian Z, Song M, Hua D and Huang Z: MicroRNA-204-5p inhibits gastric cancer cell proliferation by downregulating USP47 and RAB22A. Med Oncol 32: 331,2015 .

31. Yin Y, Zhang B, Wang W, Fei B, Quan C, Zhang J, Song M, Bian Z, Wang Q, Ni S, et al: miR-204-5p inhibits proliferation and invasion and enhances chemotherapeutic sensitivity of colorectal cancer cells by downregulating RAB22A. Clin Cancer Res 20: 6187-6199, 2014.

32. Maia J, Caja S, Strano Moraes MC, Couto N and Costa-Silva B Exosome-based cell-cell communication in the tumor microenvironment. Front Cell Dev Biol 6: 18, 2018.

33. Yáñez-Mó M, Siljander PR, Andreu Z, Zavec AB, Borràs FE, Buzas EI, Buzas K, Casal E, Cappello F, Carvalho J, et al: Biological properties of extracellular vesicles and their physiological functions. J Extracell Vesicles 4: 27066, 2015.

34. Kishore R and Khan M: More than tiny sacks: Stem cell exosomes as cell-free modality for cardiac repair. Circ Res 118: 330-343, 2016.

35. Yu DD, Wu Y, Shen HY, Lv MM, Chen WX, Zhang XH, Zhong SL, Tang JH and Zhao JH: Exosomes in development, metastasis and drug resistance of breast cancer. Cancer Sci 106 959-964, 2015

36. Lowry MC, Gallagher WM and O'Driscoll L: The Role of Exosomes in Breast Cancer. Clin Chem 61: 1457-1465, 2015.

37. Rivoltini L, Chiodoni C, Squarcina P, Tortoreto M, Villa A, Vergani B, Bürdek M, Botti L, Arioli I, Cova A, et al: TNF-related apoptosis-inducing ligand (TRAIL)-armed exosomes deliver proapoptotic signals to tumor site. Clin Cancer Res 22: 3499-3512, 2016.

38. Boelens MC, Wu TJ, Nabet BY, Xu B, Qiu Y, Yoon T, Azzam DJ, Twyman-Saint Victor C, Wiemann BZ, Ishwaran H, et al: Exosome transfer from stromal to breast cancer cells regulates therapy resistance pathways. Cell 159: 499-513, 2014.

39. Riches A, Campbell E, Borger E and Powis S: Regulation of exosome release from mammary epithelial and breast cancer cells - a new regulatory pathway. Eur J Cancer 50: 1025-1034, 2014.

40. Languino LR, Singh A, Prisco M, Inman GJ, Luginbuhl A, Curry JM and South AP: Exosome-mediated transfer from the tumor microenvironment increases TGF $\beta$ signaling in squamous cell carcinoma. Am J Transl Res 8: 2432-2437, 2016. 